\title{
Two Unusual Cases of Sudden Death in Children
}

\author{
Maria-Chiara Osterheld ${ }^{1 *}$, Marie-Hélène Perez ${ }^{2}$
}

${ }^{1}$ Institute of Pathology, University of Lausanne, Lausanne, Switzerland; ${ }^{2}$ Pediatric Intensive Care Unit, University Hospital Center and University of Lausanne, Lausanne, Switzerland.

Email: *maria-chiara.osterheld@chuv.ch

Received February $19^{\text {th }}, 2013$; revised March $19^{\text {th }}$, 2013; accepted April $15^{\text {th }}, 2013$

Copyright (c) 2013 Maria-Chiara Osterheld, Marie-Hélène Perez. This is an open access article distributed under the Creative Commons Attribution License, which permits unrestricted use, distribution, and reproduction in any medium, provided the original work is properly cited.

\begin{abstract}
We report two unusual cases of sudden unexpected death in children. Histopathologic examination showed intimal fibroplasias, a variant of fibromuscular dysplasia of the right coronary artery associated in both cases with fatty infiltration of the right ventricular myocardium. The significance of this particular combination of two lesions known to induce a sudden death in young people is discussed.
\end{abstract}

Keywords: Arrhythmogenic Right Ventricular Dysplasia-Like Cardiomyopathy; Fibromuscular Coronary Artery Dysplasia; Sudden Death

\section{Introduction}

Sudden death in children and adolescents is most often caused by either hypertrophic cardiomyopathy, arrhythmogenic right ventricular dysplasia, coronary artery anomalies, or myocarditis [1].

Fibromuscular coronary artery dysplasia (FMD) is an idiopathic non atheromatous, non inflammatory vascular disease that involves small and medium-size arteries. Intimal fibroplasias accounts for only $1 \%-5 \%$ of all cases of FMD and is characterized by primary involvement of the intimal layer of the arterial wall [2-4].

We describe here two cases of children presenting with sudden death and signs of intimal fibroplasias of the right coronary artery associated with fatty infiltration of the right ventricular myocardium.

\subsection{Case Report 1}

A nine year-old girl with mental retardation secondary to a perinatal asphyxia presented a sudden cardio-respiratory arrest and died. Neither family history nor personal history of cardiac disease was reported.

At post-mortem examination, the heart weighed 181.5 gr., which is normal for the age of the patient. The cardiac valves were normal as well as the origin of the coronary arteries. There was no increase of the epicardial fat covering the heart. The trabeculae of the right ventri-

${ }^{*}$ Corresponding author. cle appeared well developed. The thickness of the right ventricle was $0.2 \mathrm{~cm}$ and of the left ventricle $0.7 \mathrm{~cm}$. Neither major visceral pathology was detected, except the multicystic encephalopathy due to the perinatal asphyxia, nor active infection in the lungs.

Histological analysis of the heart specimens revealed an important fatty infiltration associated with moderate fibrosis in the myocardium of the right ventricle Figure 1. This fat within the myocardium extended from the subepicardial layer to intramural areas under the endocardium, sparing the left ventricle and ventricular septum. No inflammatory response was identified. The left ventricle showed neither hypertrophic fibers, nor disarray of the fibers nor adipose metaplasia. The right coronary artery displayed focal, irregular intimal thickening and a disrupted internal elastic lamina (intimal fibroplasia) Figure 2. The left coronary artery was normal.

\subsection{Case Report 2}

A nine-year-old boy with no relevant personal or familial clinical history, suffered a cardio-respiratory arrest. Resuscitation attempts were unsuccessful and the patient died.

At necropsy examination, the heart weighed 228 gr. (N: $138 \mathrm{gr})$. The cardiac valves were normal. The coronary arteries rose normally from the aorta. The thickness of the wall of the right ventricle measured $0.3 \mathrm{~cm}$ and of the wall of the left ventricle measured $1.2 \mathrm{~cm}$ revealing a 


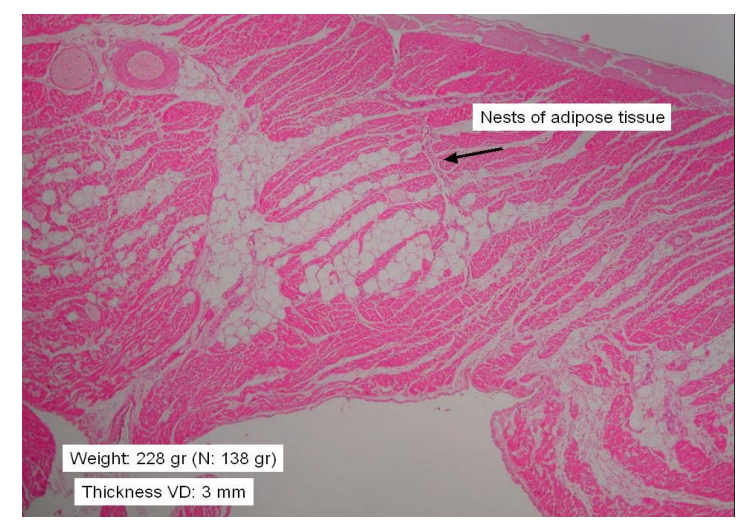

(a)

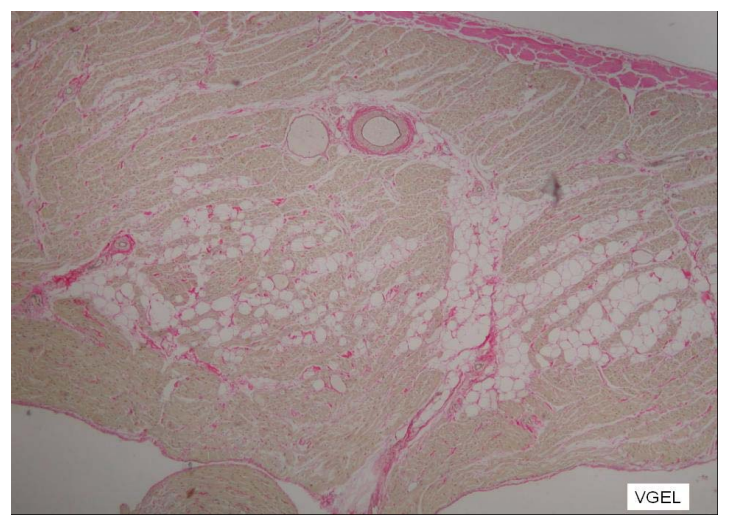

(b)

Figure 1. Histological section of the portion of right ventricule with adipose tissue. (a) Hematoxylin and Eosin stain at $\times 40$; (b) Van Gieson and Elastin stain at $\times 40$.

mild hypertrophy. Secondary changes from circulatory failure with pulmonary and cerebral oedema, renal and hepatic acute necrosis as well as focal ischemic necrosis in the large bowel were also noted.

Light microscopic examination of paraffin embedded and hematoxylin-eosin and van gieson-elastin stained sections of the heart and coronary arteries showed hypertrophic cardiomyocytes in the left myocardium. Besides, the right ventricle myocardium was totally infiltrated by fibrofatty tissue up to the sub-endocardial region without lymphocytic infiltration. The left coronary artery was normal but the right coronary artery presented an intimal thickening with an internal elastic lamina fragmentation and some disorganization of the elastic fibers in the media (intimal fibroplasia and mild medial fibromuscular dysplasia).

\section{Discussion}

The histopathological findings of both children are very similar. Both present a variant of fibromuscular dysplasia of the right coronary artery and a fatty infiltration of the right ventricular myocardium which may explain the sudden death syndrome.

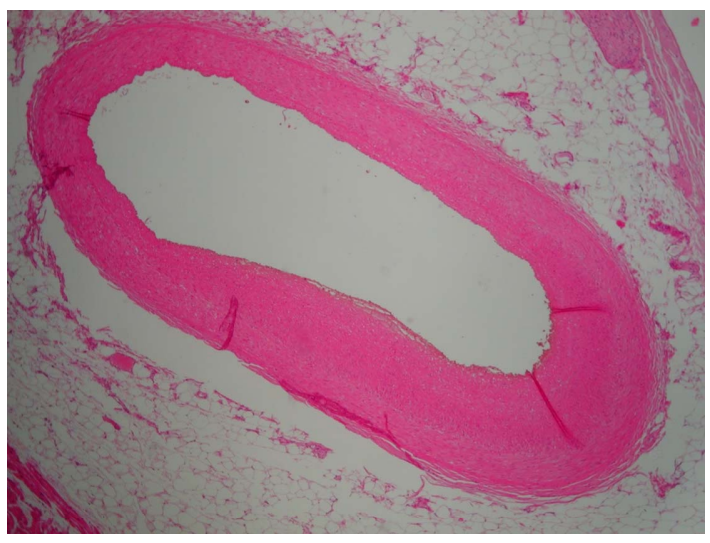

(a)

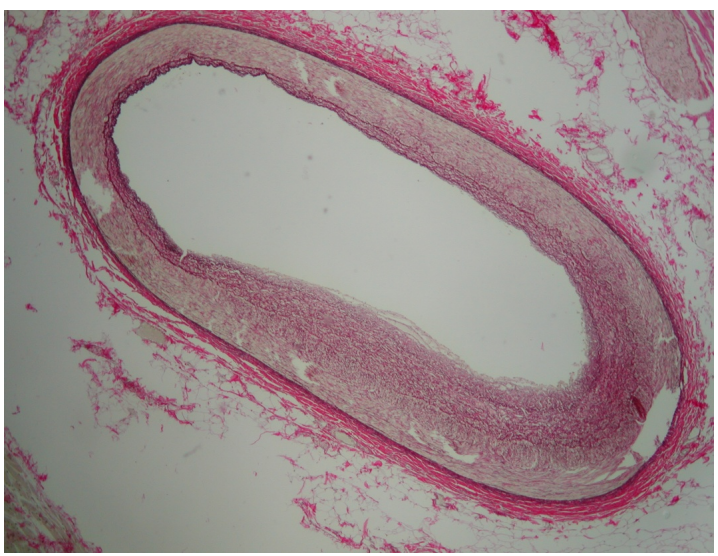

(b)

Figure 2. Histological cross-section of the right coronary artery with fibromuscular dysplasia. (a) Hematoxylin and Eosin stain at $\times 40$; (b) Van Gieson and Elastin stain at $\times 100$ and $\times \mathbf{2 0 0}$.

Arrhythmogenic right ventricular dysplasia cardiomyopathy (ARVD) is known to be a primary heart muscle disease of unknown origin characterized by transmural fatty or fibrofatty replacement of right ventricular myocardium [5]. This heart pathology represents a high risk of life-threatening ventricular electrical instability producing unexpected cardiac arrest and sudden death particularly in young people [6].

Many theories on ARVD pathobiology have been discussed in the literature. Some authors have suggested an inflammatory theory about cardiotropic viruses [7]. Others have proposed a dystrophic theory according to which myocyte death could be due to some genetic defects [8]. To support this theory some altered genes have been described [9-19]. Another possible explanation for ARVD pathobiology is ischemia. However ischemic theory has never been explored in details even if some authors have raised this hypothesis.

The presence of fat in the ventricular myocardium is a phenomenon that has been described as early as the 18th century by Senac, and Laennec [11,12]. Later, in 1850, 
Quain has described a link with clinical entities like obesity, alcoholism, sudden death and heart failure [13]. Modern literature has expanded on previous works by demonstrating that myocardial fat is not limited to ARVD but more commonly due to aging and prior myocardial infarction or chronic ischemia. Moreover, fat in the left ventricle is frequently related to prior myocardial infarction or chronic ischemia [14]. Right ventricular fat is not uncommon at necropsy, but it is usually localized in the subepicardial region only. In ARVD, the fibrofatty replacement of myocardium is transmural, as described in the right ventricle of our two patients. Because of the young age of both patients and the striking association with fibromuscular right coronary artery dysplasia, ischemia is a very likely cause of this fatty replacement of the right ventricular cardiomyocytes, and explains the fatal issue.

Fibromuscular dysplasia is a segmental non inflammatory angiopathy, originally described in 1938, as a predominantly arterial disease. It usually occurs in small and medium-sized muscular arteries and may affect the three arterial layers although the media is the most often involved. Autopsy studies suggest that the incidence within the general population is $1 \%$. The etiology remains unknown and the majority of cases are sporadic although familial forms have been described [15]. Coronary fibromuscular dysplasia has been considered as rare. A number of reports have yet documented small coronary artery FMD in patients with sudden cardiac death [16-20]. Furthermore, left main stem coronary artery FMD induces ischemic left ventricular dysfunction [21]. Ischemic degeneration as a secondary effect of coronary artery FMD is then possible. Consequently we hypothesize ischemia is a complication of the right coronary artery FMD on the right ventricle, leading fatty infiltration and sometimes fibrosis of the right myocardium. This fibrosis and fatty infiltration mimics an ARVD-degeneration of the right myocardium, which may result in a high risk of arrhythmias and sudden cardiac death.

Unfortunately, we could not perform genetic analyses in neither cases. In addition, there was no familial history of cardiac disease to support a diagnosis of RV cardiomyopathy considering the fact that ARVD has a familial occurrence in about $50 \%$ of cases, with autosomal dominant inheritance and variable penetrance as well as a polymorphic phenotypic expression [8].

These reported cases demonstrate the presence of arterial fibrodysplasia limited to one coronary artery with the coexistence of a fatty transformation of the right ventricular myocardium suggesting a possible ischemic etiology of an ARVD-like phenomenon. Therefore, in cases of sudden death in children or adolescents, a particularly careful examination of coronary arteries must be performed.
And furthermore, in patients with an ARVD confirmed by identification of pathologic mutations, the coexistence of coronary fibrodysplasia may increase the risk of cardiac arrest.

\section{REFERENCES}

[1] M. J. Davies, "The Investigation of Sudden Cardiac Death,” Histopathology, Vol. 34, No. 2, 1999, pp. 93-98. doi:10.1046/j.1365-2559.1999.00648.x

[2] K. R. Thomas, S. P. Thomas, K. O. Hewan-Lowe and J. P. Pestaner, "Fibromuscular Dysplasia in Association with Intrauterine Cocaine Exposure," Cardiovascular Pathology, Vol. 16, No. 5, 2007, pp. 313-316. doi:10.1016/j.carpath.2006.12.004

[3] M. Imamura, S. Yokoyama and K. Kikuchi, "Coronary Fibromuscular Dysplasia Presenting as Sudden Infant Death," Archives of Pathology \& Laboratory Medicine, Vol. 121, No. 2, 1997, pp. 159-161.

[4] F. Zack, H. Terpe, U. Hammer and R. Wegener, "Fibromuscular Dysplasia of Coronary Arteries as a Rare Cause of Death," International Journal of Legal Medicine, Vol. 108, No. 4, 1996, pp. 215-218. doi:10.1007/BF01369795

[5] A. Fletcher, S. Y. Ho, K. P. McCarthy and M. N. Sheppard, "Spectrum of Pathological Changes in Both Ventricles of Patients Dying Suddenly with Arrhythmogenic Right Ventricular Dysplasia. Relation of Changes to Age," Histopathology, Vol. 48, No. 4, 2006, pp. 445-452. doi:10.1111/j.1365-2559.2006.02356.x

[6] F. Fontaliran, S. Arkwright, F. Vilde and G. Fontaine, "Arrhythmogenic Right Ventricular Dysplasia and Cardiomyopathy. Clinical and Anatomic-Pathologic Aspects, Nosologic Approach,” Archives d'Anatomie et de Cytologie Pathologiques, Vol. 46, No. 3, 1998, pp. 171-177.

[7] F. Calabrese, C. Basso, E. Carturan, M. Valente and G. Thiene, "Arrhythmogenic Right Ventricular Cardiomyopathy/Dysplasia: Is There a Role for Viruses?” Cardiovascular Pathology, Vol. 15, No. 1, 2006, pp. 11-17. doi:10.1016/j.carpath.2005.10.004

[8] E. Moric-Janiszewska and G. Markiewicz-Loskot, "Review on the Genetics of Arrhythmogenic Right Ventricular Dysplasia,” Europace, Vol. 9, No. 5, 2007, pp. 259266. doi:10.1093/europace/eum034

[9] A. Rampazzo, A. Nava, G. A. Danieli, G. Buja, L. Daliento, G. Fasoli, R. Scognamiglio, D. Corrado and G. Thiene, "The Gene for Arrhythmogenic Right Ventricular Cardiomyopathy Maps to Chromosome 14q23-q24,” $\mathrm{Hu}$ man Molecular Genetics, Vol. 3, No. 6, 1994, pp. 959962. doi:10.1093/hmg/3.6.959

[10] A. Rampazzo, A. Nava, P. Erne, M. Eberhard, E. Vian, P. Slomp, N. Tiso, G. Thiene and G. A. Danieli, “A New Locus for Arrhythmogenic Right Ventricular Cardiomyopathy (ARVD2) Maps to Chromosome 1q42-q43,” Human Molecular Genetics, Vol. 4, No. 11, 1995, pp. 21512154. doi:10.1093/hmg/4.11.2151

[11] J. B. Senac, "Traité de la Structure du Coeur, de son Action et de ses Malades,” 2nd Edition, Mequignon, Paris, 1783, pp. 384-388. 
[12] R. T. H. Laennec, "Treatise on Mediate Auscultation," Vol. 2, Brosson and Claude, Paris, 1819, pp. 295-315.

[13] R. Quain, "Fatty Diseases of the Heart," Medico-Chirurgical Transactions, Vol. 33, 1850, pp. 121-152.

[14] A. H. Jacobi, A. Gohari, B. Zalta, M. W. Stein and L. B. Haramati, "Ventricular Myocardial Fat: CT Findings and Clinical Correlates," Journal of Thoracic Imaging, Vol. 22, No. 2, 2007, pp. 130-135. doi:10.1097/01.rti.0000213576.39774.68

[15] H. Suzuki, H. Daida, H. Sakurai and H. Yamaguchi, "Familial Fibromuscular Dysplasia of Bilateral Brachial Arteries,” Heart, Vol. 82, No. 2, 1999, pp. 251-252.

[16] E. Maresi, G. Becchina, G. Ottoveggio, E. Orlando, R. Midulla and R. Passantino, "Arrhythmic Sudden Cardiac Death in a 3-Year-Old Child with Intimal Fibroplasia of Coronary Arteries, Aorta, and Its Branches," Cardiovascular Pathology, Vol. 10, No. 1, 2001, pp. 43-48. doi:10.1016/S1054-8807(00)00047-8

[17] F. E. Dominguez, L. G. Tate and M. J. Robinson, "Familial Fibromuscular Dysplasia Presenting as Sudden Death,"
American Journal of Cardiovascular Pathology, Vol. 2, No. 3, 1988, pp. 269-272.

[18] K. Kaneko, T. Someya and R. Ohtaki, “Congenital Fibromuscular Dysplasia Involving Multivessels in an Infant with Fatal Outcome," European Journal of Pediatrics, Vol. 163, No. 4-5, 2004, pp. 241-244. doi:10.1007/s00431-003-1312-X

[19] A. P. Burke and R. Virman, "Intramural Coronary Artery Dysplasia of the Ventricular Septum and Sudden Death," Human Pathology, Vol. 29, No. 10, 1998, pp. 1124-1127. doi:10.1016/S0046-8177(98)90424-5

[20] K. M. Ropponen and I. Alafuzoff, “A Case of Sudden Death Caused by Fibromuscular Dysplasia,” Journal of Clinical Pathology, Vol. 52, 1999, pp. 541-542. doi:10.1136/jcp.52.7.541

[21] G. E. Pate, R. Lowe and C. E. Buller, "Fibromuscular Dysplasia of the Coronary and Renal Arteries?” Catheterization and Cardiovascular Interventions, Vol. 64, No. 2, 2005, pp. 138-145. doi:10.1002/ccd.20246 